\title{
Photobase Generator Enabled Pitch Division: A Progress Report
}

\author{
Xinyu $\mathrm{Gu}^{2}$, Younjin Cho ${ }^{1}$, Takanori Kawakami ${ }^{2}$, Yuji Hagiwara ${ }^{1}$, Brandon Rawlings ${ }^{2}$, Ryan $\mathrm{Mesch}^{1}$, \\ Toshiyuki Ogata ${ }^{1}$, Taeho Kim ${ }^{1}$, Takehiro Seshimo ${ }^{1}$, Wade Wang ${ }^{1}$, Arun K. Sundaresan ${ }^{3}$, Nicholas J. \\ Turro $^{3}$, Roel Gronheid ${ }^{4}$, James Blackwell ${ }^{5}$, Robert Bristol ${ }^{5}$, C. Grant Willson ${ }^{1,2}$ \\ ${ }^{I}$ Department of Chemistry and ${ }^{2}$ Department of Chemical Engineering, The University of Texas at \\ Austin, TX 78712, USA; ${ }^{3}$ Department of Chemistry, Columbia University, New York, NY 10027, USA; \\ ${ }^{4}$ IMEC, Leuven, Belgium; ${ }^{5}$ Intel Corporation, Hillsboro, OR 97124, USA;
}

\begin{abstract}
Pitch division lithography (PDL) with a photobase generator (PBG) allows printing of grating images with twice the pitch of a mask. The proof-of-concept has been published in the previous paper[1, 2] and demonstrated by others[1]. Forty five nm half-pitch (HP) patterns were produced using a 90nm HP mask, but the image had line edge roughness (LER) that does not meet requirements. Efforts have been made to understand and improve the LER in this process. Challenges were summarized toward low LER and good performing pitch division. Simulations and analysis showed the necessity for an optical image that is uniform in the $\mathrm{z}$ direction in order for pitch division to be successful. Two-stage PBGs were designed for enhancement of resist chemical contrast. New pitch division resists with polymer-bound PAGs and PBGs, and various PBGs were tested. This paper focuses on analysis of the LER problems and efforts to improve patterning performance in pitch division lithography.
\end{abstract}

Keywords: pitch division, photobase generator, PBG, polymer bound PAG, polymer bound PBG, two-stage PBG, non-uniform image, acid diffusion, base diffusion

Advances in Resist Materials and Processing Technology XXVIII, edited by Robert D. Allen, Mark H. Somervell, Proc. of SPIE Vol. 7972, 79720F · (C) 2011 SPIE · CCC code: 0277-786X/11/\$18 · doi: 10.1117/12.879861 


\section{Introduction}

Delays in readiness of EUV lithography force the semiconductor industry to explore new technologies based on the existing methods to enable the patterning at $32 \mathrm{~nm}$ and $22 \mathrm{~nm}$ nodes. An economic and efficient way to assist the current lithographic methods to achieve higher resolution than the theoretical limit is highly demanded. Double patterning lithography (DPL) and self-aligned double patterning (SADP), two resolution enhancement techniques, show great promise as approaches to accessing the small features required for next generation microprocessors. Unfortunately, both techniques require extra processing steps, which increase the cost-of-ownership (CoO). We hope to develop a technique that would provide access to the $22 \mathrm{~nm}$ and $16 \mathrm{~nm}$ nodes, which does not require extra processing steps or new capital equipment. A material based pitch division lithography proposed by our group in 2009[2] would fulfill that goal. The pitch division technique requires only a single exposure to create pitch divided patterns without extra processing steps. The only difference between this approach and the conventional lithography is that the resist formulation manifests a dual-tone behavior instead of just a positive tone or a negative tone response. The dual-tone is achieved by including both a photoacid generator (PAG) and a photobase generator (PBG) into the resist polymer formulation. The details of this concept are described in the early papers[2,3].

The PBG generates a base upon UV irradiation just like PAG generates acid. In the resist matrix, base molecules and acid molecules react with each other and are neutralized, resulting in a net mount of acid or base and a corresponding salt. The salt is considered to be inert. In the case of slower base generation rate $\left(\mathrm{k}_{\mathrm{b}}<\mathrm{k}_{\mathrm{a}}\right)$ and higher PBG loading, the net amount of acid produced as a function of exposure dose is parabola like in form. These particular acid generations kinetics can produce a dual tone response. In order to successfully capture the dual tone response, the image contrast needs to be higher than the E-factor which was defined in the previous paper[3] as $\left(E_{n}-E_{0}\right) /\left(E_{n}+E_{0}\right)$.

Pitch divided line and space (L/S) patterns have been achieved by this approach on an ArF scanner $(\mathrm{NA}=0.85)$. The following SEM images show the pitch divided gratings printed with a commercial resist blended with a photobase generator. As can be seen, pitch division is achieved at different pitch sizes down to $45 \mathrm{~nm}$ HP. This is a process latitude factor $\left(\mathrm{k}_{1}\right)$ of 0.20 , which is smaller than the theoretical limit of 0.25 . If this approach could be made to work at a manufacturing level, the capability of the current lithographic process could be extended without increasing the production cost. However, severe line edge roughness (LER) was observed with the dual-tone resist across the entire exposed field. There is bridging and footing in the trenches as well. The patterning performance is not satisfactory as the LER clearly exceeds the ITRS tolerance $(3 \sigma<8 \% \mathrm{CD})[4]$.

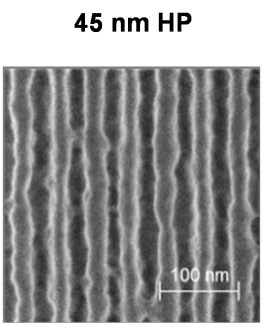

$50 \mathrm{~nm} \mathrm{HP}$

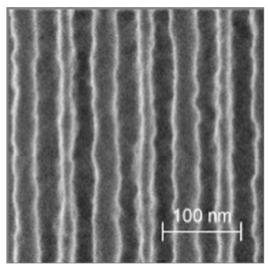

$60 \mathrm{~nm} \mathrm{HP}$

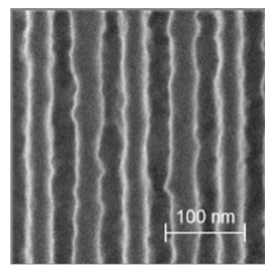

75 nm HP

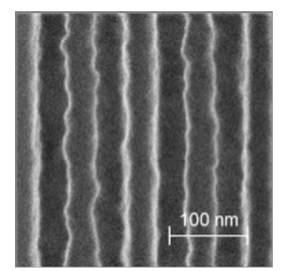

Figure 1. Pitch division pattern was achieved with various pitch sizes. The resist formulation was a commercial resist with addition of $5.5 \mathrm{mM}$ dicyclohexyl o-nitrobenzyl carbamate. (NA=0.85, PSM w/ 6\% Attn., X-dipole, etc.) 
The continuing research efforts have been focused on reducing the LER and enlarging the lithographic process window for pitch division. Solving these issues requires a good understanding of the PD process and how its patterning performance is related to the processing parameters and the photo responses. The patterning performance is directly determined by the optical image contrast and the dissolution contrast of the resin at the both dose thresholds. However, this is different from the conventional resist case in various ways. First, the line edges are defined by the top and bottom regions of the sinusoidal image as opposed to the center region where the image slope is the highest for the conventional resist. Secondly, the net acid generation for the dual-tone resist is a parabola like function rather than the conventional first order exponential decay. Last but not least, defocusing in the resist affects two tones simultaneously and more effectively than it does in a conventional resist. We postulate that it is these unique behaviors of the dual tone resist that are responsible for the poor LER. There are three major challenges associated with these unique behaviors, which need to be addressed to achieve pitch division with low LER. Those challenges are listed below.

1) Minimize the non-uniformity of the image-in-resist along the $Z$ direction

2) Maximize the slope of the acid generation curve across the threshold dose

3) Limit the diffusion of photochemically produced acid and base

Simulations and experiments were designed and performed to provide guidance for addressing these challenges. The results will be described in this paper.

\section{Z-directional Non-uniform Image-in-resist}

At high numerical aperture (NA) lithography, the relative amount of TE versus TM polarization increases as the diffraction angle goes higher, degrading the image quality[5]. Multi-layer BARC has been proven to further reduce the substrate reflectance and thereby improve the image quality, but this is not within the scope of this paper. We explore the capability of this pitch division resist based only on a typical single-layer BARC.

A single-layer BARC has been used in the photolithography process for years to reduce substrate reflectance. Even though some trace amount of substrate reflectance is inevitable with single-layer BARCs, the resulting standing wave and swing curve do not affect the imaging of conventional resist greatly but can cause trouble in imaging the dual-tone resist. Simulations were performed to demonstrate this point and are described below.

The resist profiles after development were simulated for a given image-in-resist for both positive resist and dual-tone resist. The simulation strategy is to keep as many processing parameters constant as possible for both positive and dual-tone resists, except the following: acid generation kinetics, incident exposure dose and PEB temperature. It is obvious that the acid generation kinetics is different as in the dual-tone resist it is net acid that is important and this involves an additional base generation process. The incident exposure dose was varied to target a 1:1 L/S and is the sole variable in the single-tone simulation. The dual-tone imaging involves two sets of line widths, which cannot be tuned independently through any processing variable. Thus, both the incident exposure dose and the PEB temperature were 
used to adjust the positive and negative line widths to achieve a 1:1 L/S ratio. The simulation for positive tone resist followed a single run PROLITH simulation routine with a dose-to-size iteration whereas that for the dual-tone case was performed with a customized MATLAB code. The code was developed based on the prototype modeling code described in this paper[6]. The code makes use of MATLAB-PROLITH programming interface (PPI) to realize output of image-in-resist, execution of customized acid generation, input of acid image into PROLITH, and simulation of the final resist profile. The code is also able to analyze the dual-tone resist profile, generating CDs for both positive and negative lines. The CD error was fed back to the commander and another simulation run was made with a different combination of exposure dose and PEB temperature until the target 1:1 L/S is reached.
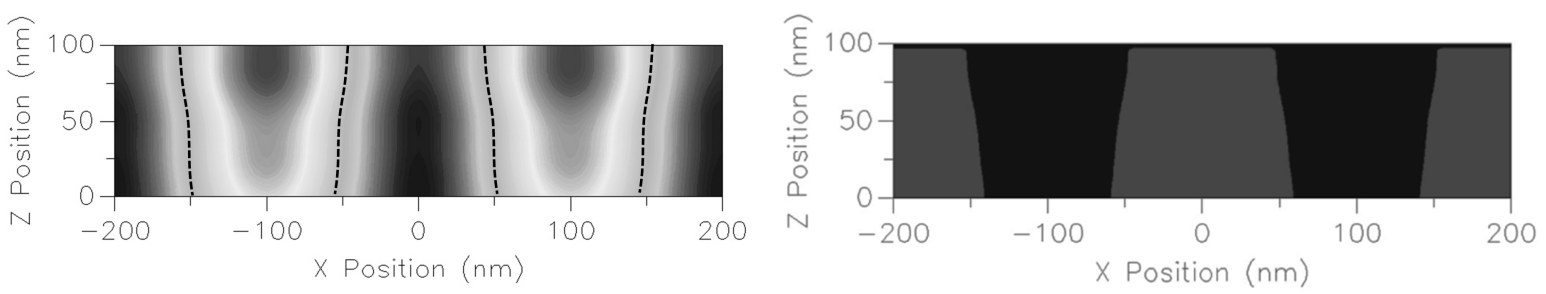

Figure 2. The left image is a typical optical image in the resist produced by the following illumination condition $\left(\mathrm{NA}=0.85, \mathrm{X}\right.$-Dipole, $100 \mathrm{~nm} \mathrm{~L} / \mathrm{S}$ PSM w/ 6\% Attn., $\mathrm{n}=1.736$, Dill B $=3.08 \mu \mathrm{m}^{-1}$,

$\mathrm{BARC}(\mathrm{ARC} 29 \mathrm{~A})=84 \mathrm{~nm})$; The right image is the simulated $\mathrm{x}$-section profile for a positive tone resist.

Figure 2 shows a typical optical image in the resist film under certain illumination condition. The illumination parameters are described in the caption. The resist profile of single positive tone exposure was simulated using PROLITH and shown in Figure 2. It can be seen from the input image-in-resist that there exists a low extent of standing wave and certain absorbance. The resulting resist profile for the positive tone is still quite acceptable. The side wall angle is slightly reduced due to the absorbance but the pattern is satisfactory for the next etching step. Yet, the patterning of dual-tone resists is different. The cross-section profile is defined by the high intensity and low intensity region in the image-in-resist. Standing waves have the greatest effect on the high intensity end. This can also be seen in the stacked intensity profiles shown in Figure 3. There is greater intensity variation near the intensity peak, directly leading to a poor $\mathrm{CDU}$ in both the $\mathrm{XY}$ direction and $\mathrm{Z}$ direction. The simulated pitch division profile is shown in Figure 4 . As can be seen, the negative tone lines undercut, reproducing the shape of the high intensity portion of the image-in-resist, whose shape is largely determined by the absorption. It is necessary to mention that the negative lines are more sensitive to perturbation than the positive lines as observed from simulation results. The bottom of the negative lines tends to disappear, resulting in peeling off or "adhesion loss" of the negative lines. This greatly reduces the exposure latitude for the negative images. 

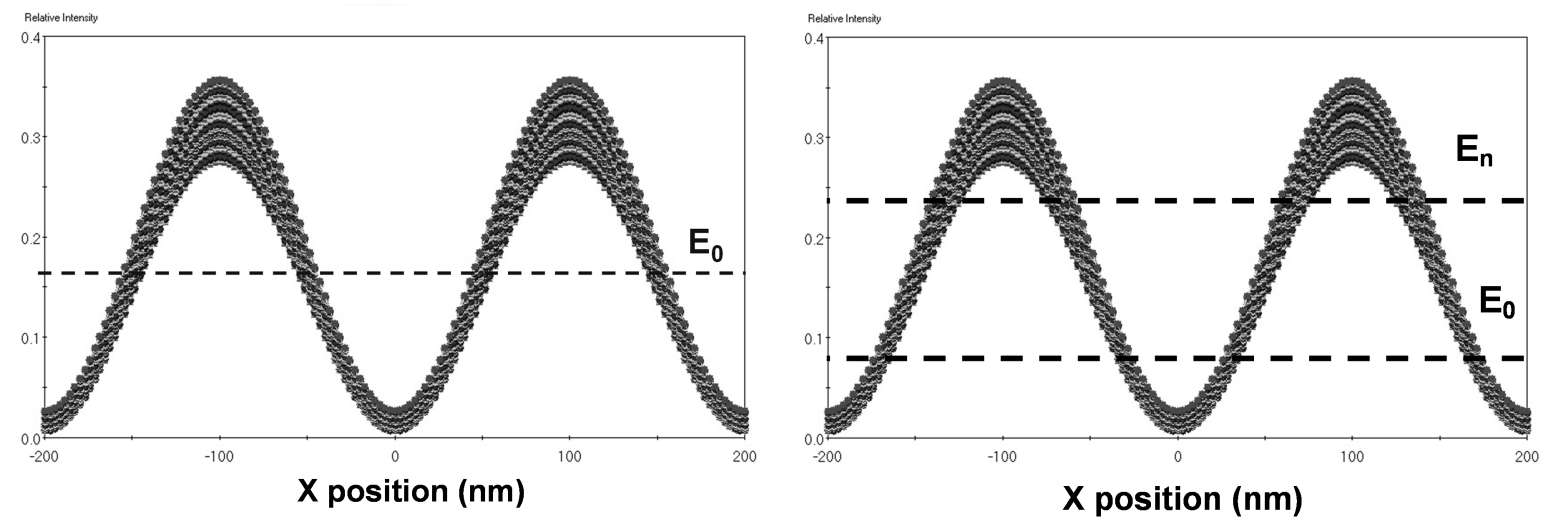

Figure 3. Stacked z-directional intensity profile (light intensity profiles at each z position in the image-in-resist shown in Figure 2). This plot shows the intensity variance along the z-direction in the image-in-resist.
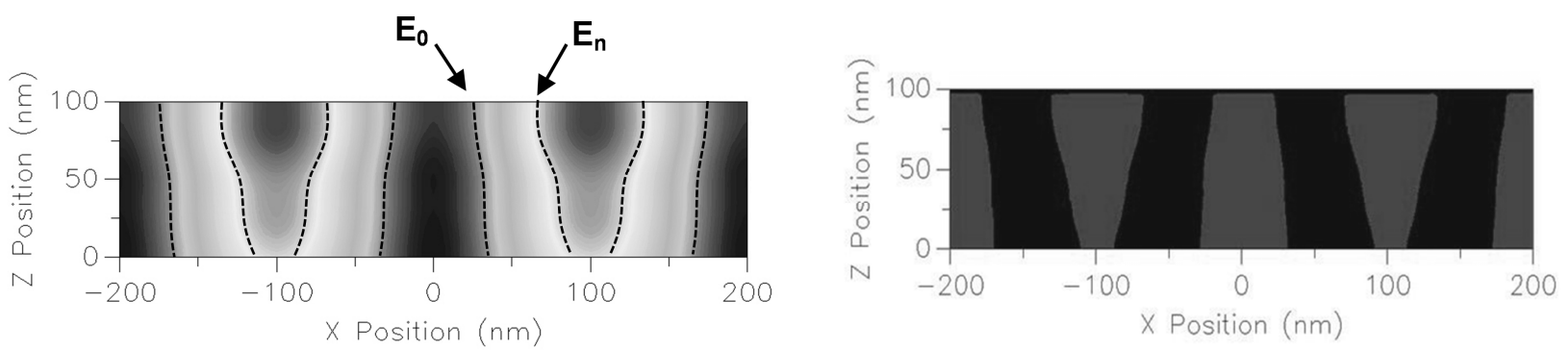

Figure 4. The left image shows the image-in-resist with two sets of dashed lines, indicating how the positive tone lines and the negative tone lines are defined by $\mathrm{E}_{0}$ and $\mathrm{E}_{\mathrm{n}}$. The right image is the simulated cross-section profile for pitch division profile.

If there is a BARC thickness error, the standing wave issue becomes even worse as seen in Figure 5. The positive resist is still able to resolve the image and produce a fair pattern profile. However, when this image-in-resist is applied to a dual-tone resist, the simulation shows a poor negative tone profile due to the standing wave. The "neck" in the negative line is produced by the minimal intensity node of the standing wave, as illustrated in Figure 6. This portion of the resist pattern is very weak and tends to mechanically break during the development process. The SEM image (Figure 7) illustrates an example, showing failure of all the negative lines. Those negative tone lines are still insoluble but unable to survive the development process. They break off due to the extremely thin bottom cross section. Some residue of the failed negative lines can still be observed in the trenches. 

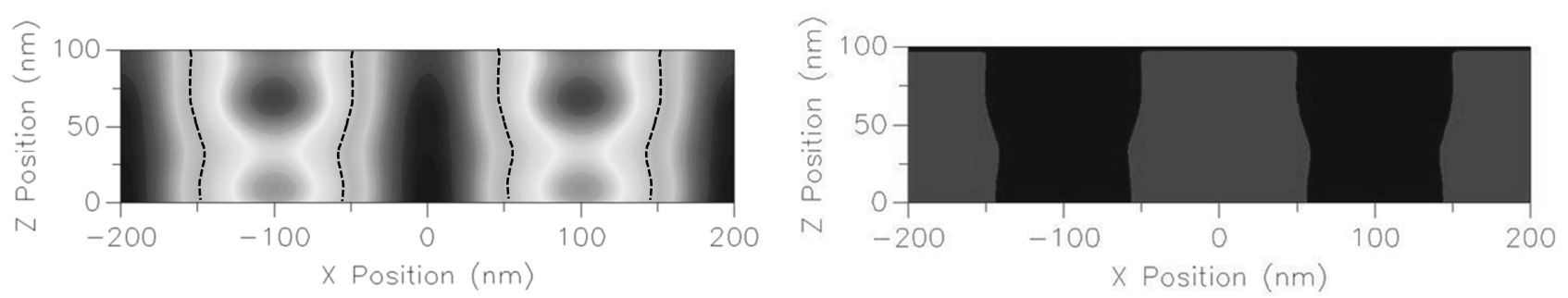

Figure 5. Image-in-resist when BARC thickness is 5\% thinner than the optimized. The dashed shows the positive tone dose threshold. The right image is the simulated resist profile.
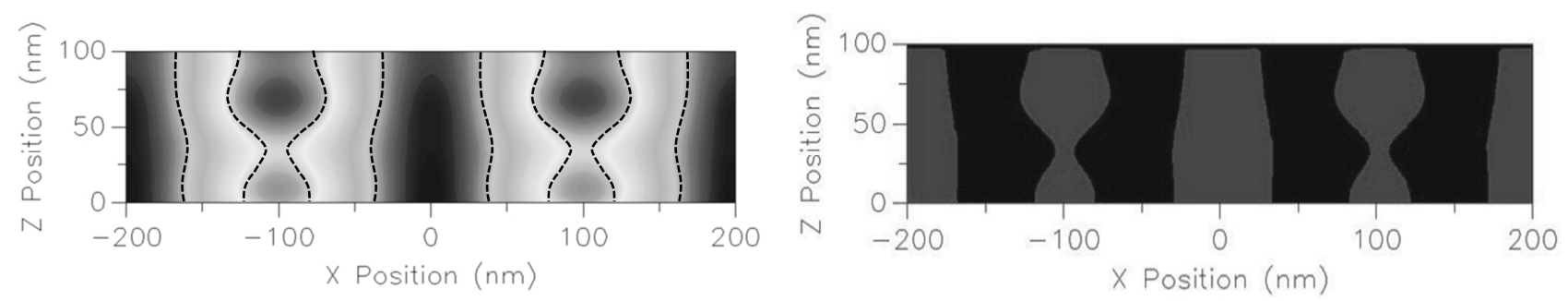

Figure 6. In the case of dual-tone patterning, the En is defined in the high intensity area whereas the E0 is defined by the dark end of the optical image. The right image is the simulated pitch division profile.

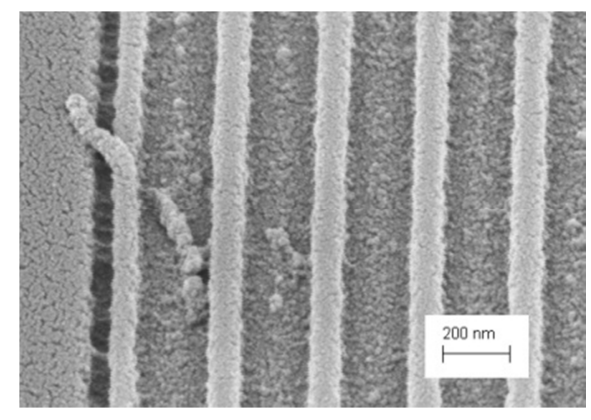

Figure 7. SEM image of a dual-tone resist printed with poor image-in-resist

The implication of these observations and the analysis is that in order to solve the poor negative tone performance the standing wave and the absorption needs to be reduced. We must maximize z-directional uniformity in the image-in-resist. One way to achieve this is to reduce the resist film thickness. Thinner resist films result in lower total film absorbance, which will improve the side wall angle. Also, when the resist layer is thinner than the standing wave, only part of the wave influences the standing wave. Figure 8 shows the PROLITH simulation of image-in-resist with varying the resist film thickness. Assuming the optical focus is on the resist surface, the uniformity of the image-in-resist improves as the resist thickness is decreased. The stacked intensity profiles are also plotted and show a much smaller variation at the high intensity region of the optical image, thereby improving LER in the negative tone lines. 


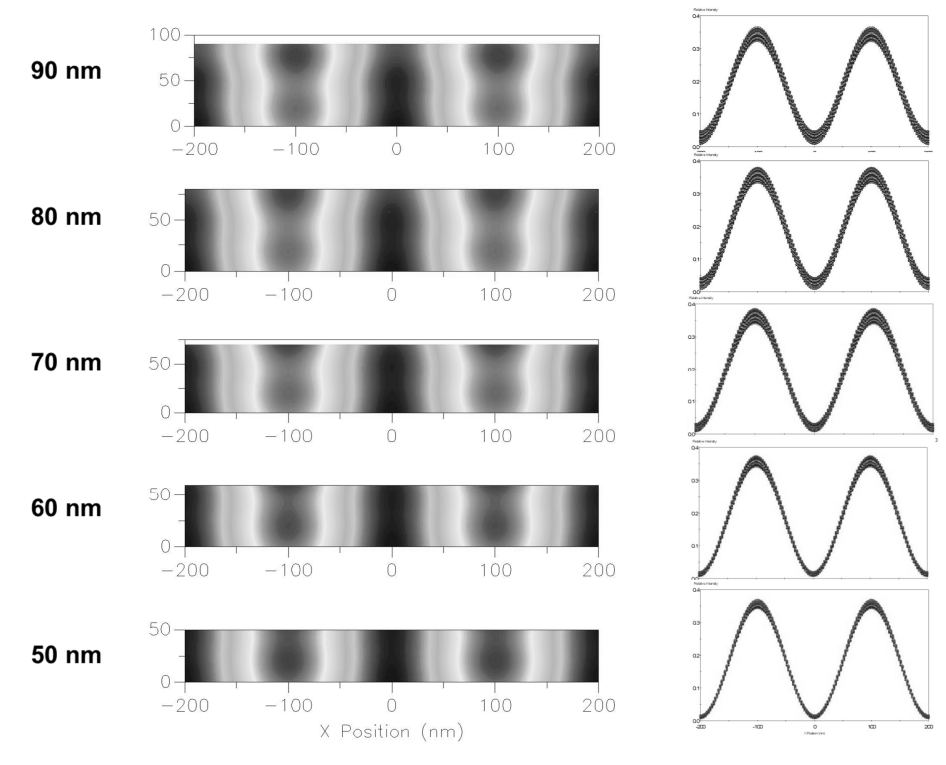

Figure 8. Influence of resist film thickness on the image-in-resist and the stacked intensity profile

The resist profile was simulated with a $50 \mathrm{~nm}$ thin resist film by PROLITH using the same PPI code. The side wall angles for both positive and negative lines are greatly improved as shown in Figure 8. Hence, the process window for pitch division is enlarged accordingly and can be further improved in conjunction with the methods described in the next section. There are several concerns about the thin resist film. These include acid loss through the resist surface and changes in resist physical properties[7]. These concerns will be considered during the lithographic evaluation with thin resist films $(\sim 50 \mathrm{~nm})$ that will be carried out in the future.

\section{Improve Chemical Contrast}

The chemical contrast in the resist greatly affects line edge roughness. The chemical contrast is solely the acid gradient in the scope of this paper. Compared to conventional ArF resists, the PBG based dual-tone resists have lower chemical contrasts mainly because the base generation slows down and ultimately shuts off the net acid production. If one ignores the optical image contrast and merely considers the dose dependent acid generation slope at the thresholds, it is clear that the acid generation slope in a conventional resist can be expressed as equation (1), whereas for the PDL resist, equation (2) adds a base generation term, which is in negative value, degrading the acid generation slope. Figure 9 illustrates the higher acid generation slop from the conventional acid generation.

$$
\begin{gathered}
\frac{d[\text { Acid }]_{\text {conv }}}{d D}=k_{a} \cdot e^{-k_{a} D} \\
\frac{d[\text { Acid }]_{d u a l}}{d D}=k_{a} \cdot e^{-k_{a} D}-k_{b} \cdot e^{-k_{b} D}
\end{gathered}
$$

$\mathrm{k}_{\mathrm{a}}$ and $\mathrm{k}_{\mathrm{b}}$ in the equations are the kinetic constants and $\mathrm{D}$ is the exposure dose applied to the resist. In these equations, the 
PAG loadings for both resists are normalized to the same value to ensure a valid comparison between the kinetics.

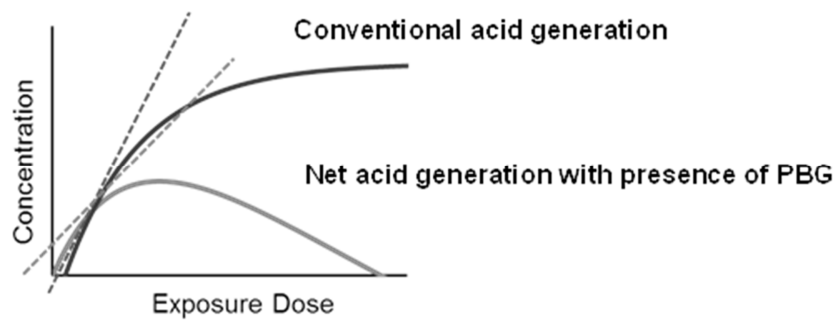

Figure 9. Comparison between conventional acid generation kinetics and net acid generation in dual-tone resists.

Degradation in chemical contrast due to the addition of a photobase generator could, to large extent, be responsible for the line edge roughness, especially the high frequency LER. Clearly it is important to improve the chemical contrast to reduce LER. It can be imagined that a delay in generating base would maintain the chemical contrast at the same level as that of a conventional resist at the positive end; and onset of rapid base generation after certain amount of exposure would create a sharp drop in acid generation, producing a high contrast negative tone. Figure 10 (b) shows a cartoon example of this sort of base generation kinetics.
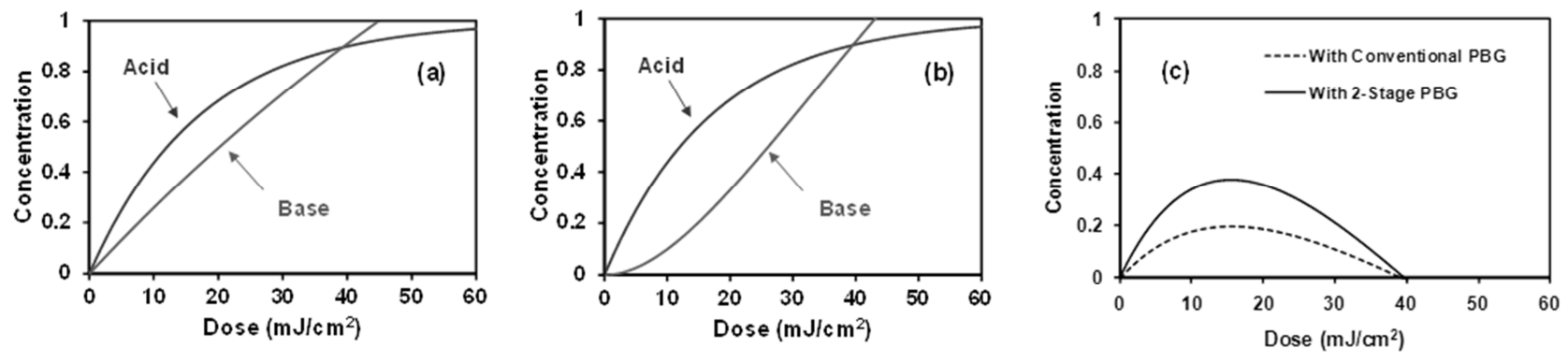

Figure 10. (a) Acid and base generation from a regular dual-tone resist; (b) A base generation with self-acceleration produced by two-stage PBG; (c) Comparison of net acid production curve between conventional PBGs and two-stage PBGs;

There is no chemical analog of photochemical base generation of the sort described above in the existing literature, but this sort of kinetic function can be achieved if there is a slow increase in base concentration at the beginning of the exposure followed by a rapid increase in rate with conversion. Kinetics of this sort can be realized by a series reaction shown below.

$$
L-P B G \stackrel{h v}{\longrightarrow} P B G \stackrel{h v}{\longrightarrow} \text { Base }
$$

Here, the base generation is executed in a linked, two-stage reaction. The photobase generation is plotted in Figure 10 (b). It can be seen that the base generation accelerates in the beginning stage of the reaction. To fully utilize this portion of the base generation curve, the PBG loading needs to be higher than the PAG loading. The resulting net acid generation can then be compared to that from the regular PBG, shown in Figure 10 (c). With the same amount of PBG and PAG loading, the chemical contrast with the two-stage PBG is superior. 


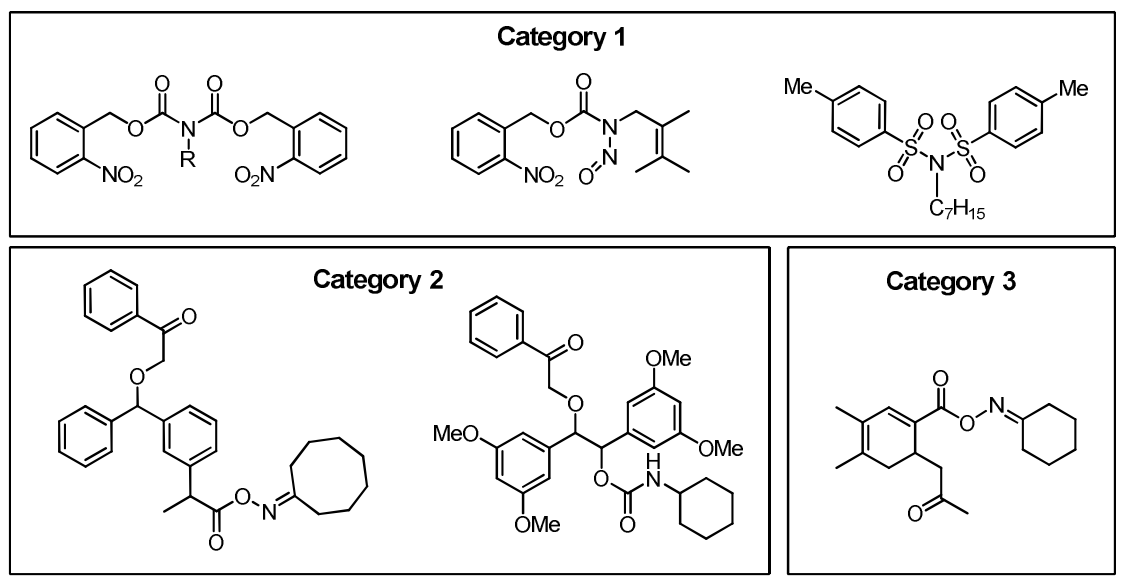

Figure 11. Structures of designed two-stage PBGs devided into three categories in terms of base generation mechanism

With the guidance from the above analysis, three types of two-stage PBG were designed. Figure 11 shows examples from each category. The first category is a difunctional photolabile base generator. The latent PBG is an amine protected by two photolabile functional groups. The possible photolabile groups include the o-nitrobenzyl carbamoyl group, toluenesulfonyl group and nitroso group. In these systems, it takes two consecutive photo-cleavage reactions to release the amine. The second category is based on the PBGs with protected photosensitizers, e.g. benzophenone and tetramethoxy benzoin. Upon irradiation, the first photochemical cleaves the protecting group (phenyl acetyl), which releases a triplet photosensitizer for the reaction that produces an amine. The third category involves PBGs with latent aromatic rings. A PBG of this sort undergoes a Norrish type II cleavage of the protecting group with the first photon, producing an aromatic ring. The substance, thereby, becomes more absorbing at $193 \mathrm{~nm}$, which enables the base generation by the second photochemical process. We have very recently completed the synthesis of some of these target two-stage PBGs. Synthesis strategies and photophysical studies will be reported in future papers.

\section{Diffusion of Acid and Base}

Diffusion of photo acid has been indicated as a cause for LER in ArF and KrF resists and has been extensively studied in the lithography field [8-11]. In the early stage of our study, acid and base diffusion was thought to be one of the major contributers to the LER observed with the $45 \mathrm{~nm}$ HP PD patterns and might be also the limiting factor preventing this PD approach from achieving smaller features. We therefore set out to limit the acid and base diffusion by covalently attaching the PAG and PBG to the resist polymer backbone. This approach to controlling mass transport has been used by many others[12-14]. Our purpose was to compare the non-bound and bound cases and verify whether the major source of the observed LER is diffusion of acid and base. In a separate paper [7972-71], the synthesis and characterization of polymer bound PAGs and PBGs is described in detail. 

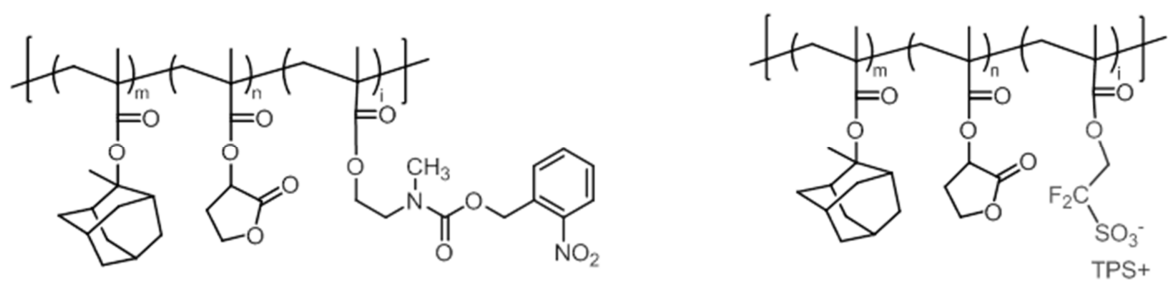

Figure 12. Structures of polymer bound PAG and PBG

The structures of these polymers are shown in Figure 12. The polymer bound PAG, in particular, might be much less sensitive than non-bound PAG. The corresponding photo acid is bound to the backbone. During PEB, the bound acid would affect less area and catalyze less number of deprotection reactions. This is indeed what we observed with polymer bound PAG in the contrast curve measurement. Figure 13 (a) shows a contrast curve with the polymer bound PAG. When the two dose thresholds are getting closer through formulation optimization, at some level the soluble film in the medium dose region eventually becomes partially insoluble as indicated by the undissolved film in the medium dose region. Under the same condition with non-bound PAG, the resist film exposed with medium dose is still soluble. Hence, the polymer bound PAG was not further investigated but the polymer bound PBG was studied and compared to the non-bound case.

To study the effect of attaching PBG to the polymer backbone, two resist formulations were prepared in the way that the only difference between the two is intended to be whether PBG is bound to polymer backbone. However, the composition of GBLMA and MAdMA in the polymer bound PBG might not be exactly the same as those in the non-bound resin. Also, incorporation of PBG monomer into the polymer backbone might change the dissolution properties of the resin. Thus, the loadings of each component were slightly adjusted to produce similar E-factors as the goal.
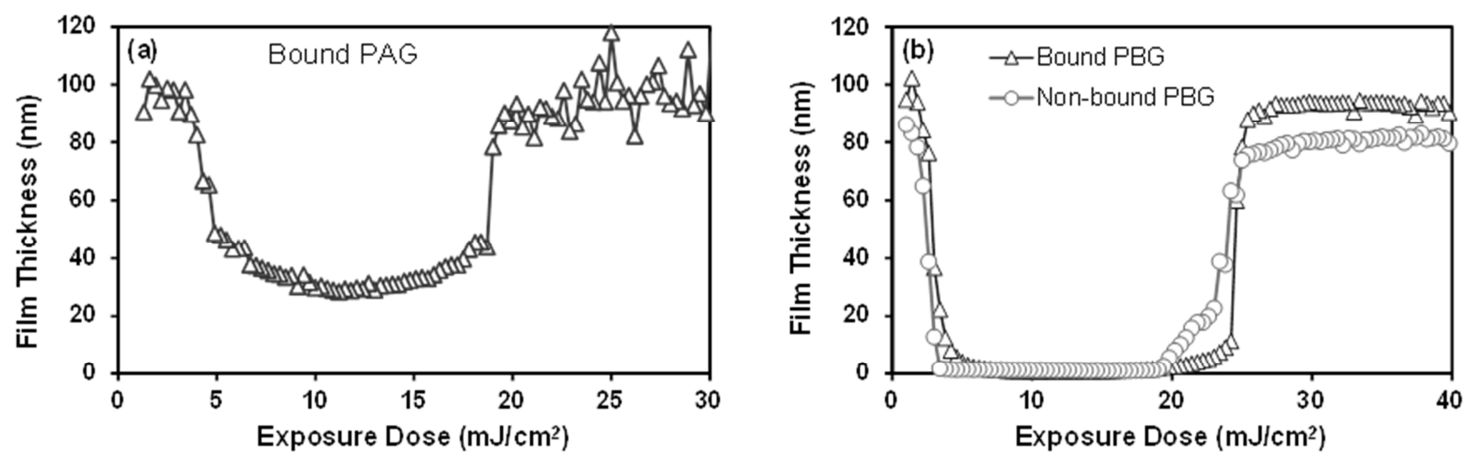

Figure 13. (a) Contrast curve of dual-tone formulation with polymer bound PAG; (b) Contrast curves of dual-tone formulation with polymer bound PBG and non-bound PBG;

Figure 13 (b) shows the contrast curves from the two formulations which have similar E-factors. The contrast of negative tone response is sharper with the polymer bound PBG. The two formulations were then subjected to lithographic evaluations. The exposure tests were performed with a $300 \mathrm{~nm}$ pitch 1:1 L/S binary mask under NA of 0.85 . The 
exposure dose was varied and the results are shown in Figure 14. Although microbridging is severe in both cases, pitch division was observed as indicated by the $150 \mathrm{~nm}$ pitch size, which is half of that on the mask. Clearly, the polymer bound PBG provides a wider exposure latitude $(\sim 10 \%)$ for producing a pitch division pattern, as indicated by the box in Figure 14. Under these conditions, the non-bound PBG formulation barely produces pitch division. We are assuming the unreacted PBG and released base on the polymer backbone do not enhance the dissolution contrast of the resist polymer. This is an assumption that needs to be verified in the future, but limiting base diffusion is clearly while advantageous to some extent, it is not the cure for the LER problem.

(a)
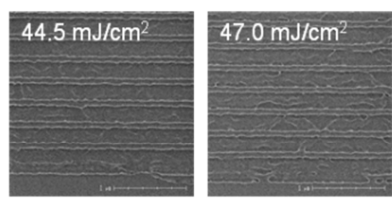

$49.5 \mathrm{~mJ} / \mathrm{cm}$

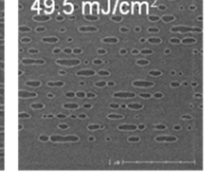

(b)
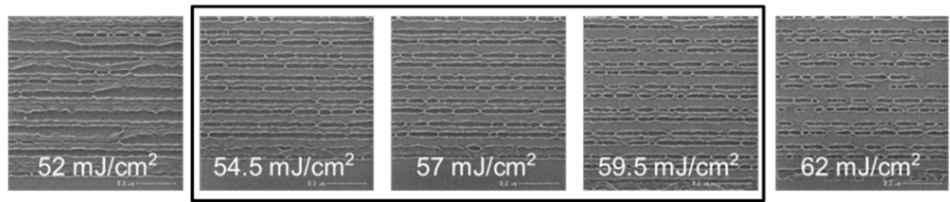

Figure 14. Lithographic evaluation: (a) non-bound PBG formulation; (b) Polymer bound PBG formulation. The two formulations produce similar contrast curves, similar E-factor, as shown in Figure 13 (b)

\section{Conclusions and Future Work}

We reported a pitch division technique that makes use of a photobase generator enabled dual-tone resist to extend the resolution capability of the current lithographic method with no additional cost. More patterning results using a commercial scanner were shown in this paper. Pitch division was successfully achieved at various pitch sizes with a NA of 0.85 and a $k_{1}$ of 0.20 was obtained. However, poor line edge roughness is still a challenge and takes the form of microbridging and footing. Efforts have been focused on understanding and improving LER.

Three major contributors to the LER were identified and appropriate solutions were proposed. Firstly, PDL requires an optical image in resist that is uniform in the $\mathrm{z}$ direction to ensure straight side walls for both positive and negative tone lines. A thinner resist layer and very tight control of the BARC thickness were proposed to achieve z-uniformity through minimizing both standing waves and total film absorbance. Secondly, the chemical contrast being degraded by addition of PBG; the dual-tone resist possesses lower chemical contrast than conventional resists. Two-stage PBGs were proposed to improve the chemical contrast for dual-tone resists at both positive and negative tone ends. The synthesis of two-stage PBGs is ongoing and will be presented in the future. Thirdly, diffusion of acid and base might be responsible for the LER in the dual-tone resist. Limiting diffusion by attaching the photoacid and photobase generating groups to polymer backbones was achieved. The pattern quality was not improved as much as expected, but through limiting the base diffusion, a larger exposure latitude for pitch division was observed. Currently, lithographic evaluations of pitch division formulations are in progress under the guidance of the analysis described in this paper. Efforts are also focused on the synthesis and characterization of two-stage PBGs and their associated photophysical measurements. 


\section{ACKNOWLEDGEMENT}

The authors would like to thank Intel Corporation for funding, Shang-ho Lin and Brian Osborn at JSR for their help in lithographic evaluation, Alex Vaglio at IMEC for help with SEM, Stephen Golledge at CAMCOR for surface analysis, Chris Mack and many others. The authors would also like to acknowledge KLA-Tencor for providing academic licenses for PROLITH software at UT.

\section{REFERENCE}

[1] J. Hatakeyama, M. Ohashi, Y. Ohsawa, K. Katayama, and Y. Kawai, "Inactivation Technology for Pitch Doubling Lithography,” Journal of Photopolymer Science and Technology, 23(2), 251-258 (2010).

[2] X. Gu, C. Bates, Y. Cho, E. Costner, F. Marzuka, T. Nagai, T. Ogata, C. Shi, A. K. Sundaresan, N. J. Turro, R. Bristol, P. Zimmerman, and C. G. Wilson, “A New Materials-based Pitch Division Technique,” Journal of Photopolymer Science and Technology, 22(6), 773-781 (2009).

[3] X. Gu, C. M. Bates, Y. Cho, T. Kawakami, T. Nagai, T. Ogata, A. K. Sundaresan, N. J. Turro, R. Bristol, P. Zimmerman, and C. G. Willson, "Photobase Generator Assisted Pitch Division," Proc. of SPIE, 7639, 763906 (2010).

[4] D. J. C. Herr, "The Potential Impact of Natural Dopant Wave front Roughness on High Frequency Line Edge Roughness Requirements," International Technology Roadmap for Semiconductors, 694 (2005).

[5] J. B. Claypool, M. Weimer, V. Krishnamurthy, W. Gehoel, and I. S. K. van, "New advanced BARC materials for ultra-high NA applications," Proc. SPIE-Int. Soc. Opt. Eng., 5753(Pt. 2, Advances in Resist Technology and Processing XXII), 679-689 (2005).

[6] S. Lee, K. Jen, C. G. Willson, J. Byers, P. Zimmerman, and N. J. Turro, "Materials modeling and development for use in double-exposure lithography applications," J. Micro/Nanolith. MEMS MOEMS, 8(1), 011011 (2009).

[7] A. Sundaramoorthi, T. R. Younkin, and C. L. Henderson, "Elucidating the physiochemical and lithographic behavior of ultra-thin photoresist films." 7273, 72733V (2009).

[8] F. A. Houle, W. D. Hinsberg, M. Morrison, M. I. Sanchez, G. Wallraff, C. Larson, and J. Hoffnagle, "Determination of coupled acid catalysis-diffusion processes in a positive-tone chemically amplified photoresist," Journal of Vacuum Science \& Technology B, 18(4), 1874-1885 (2000).

[9] J. Nakamura, H. Ban, K. Deguchi, and A. Tanaka, "EFFECT OF ACID DIFFUSION ON RESOLUTION OF A CHEMICALLY AMPLIFIED RESIST IN X-RAY-LITHOGRAPHY,” Japanese Journal of Applied Physics Part 1-Regular Papers Short Notes \& Review Papers, 30(10), 2619-2625 (1991).

[10] K. Natsuda, T. Kozawa, K. Okamoto, and S. Tagawa, "Study of acid-base equilibrium in chemically amplified resist," Japanese Journal of Applied Physics Part 1-Regular Papers Brief Communications \& Review Papers, 46(11), 7285-7289 (2007).

[11] M. Yoshizawa, and S. Moriya, "Study of the acid-diffusion effect on line edge roughness using the edge roughness evaluation method," Journal of Vacuum Science \& Technology B, 20(4), 1342-1347 (2002).

[12] M. D. Stewart, H. V. Tran, G. M. Schmid, T. B. Stachowiak, D. J. Becker, and C. G. Willson, “Acid catalyst 
mobility in resist resins," Journal of Vacuum Science \& Technology B, 20(6), 2946-2952 (2002).

[13] R. D. Allen, P. J. Brock, Y.-H. Na, M. H. Sherwood, H. D. Truong, G. M. Wallraff, M. Fujiwara, and K. Maeda, "Investigation of polymer-bound PAGs: synthesis, characterization and initial structure/property relationships of anion-bound resists," Journal of Photopolymer Science and Technology, 22(1), 25-29 (2009).

[14] M. X. Wang, N. D. Jarnagin, C. T. Lee, C. L. Henderson, W. Yueh, J. M. Roberts, and K. E. Gonsalves, "Novel polymeric anionic photoacid generators (PAGs) and corresponding polymers for $193 \mathrm{~nm}$ lithography," Journal of Materials Chemistry, 16(37), 3701-3707 (2006). 